\title{
Stability of a plane soliton to infinitesimal two-dimensional perturbations
}

\author{
P. G. Saffman \\ Department of Applied Mathematics, California Institute of Technology, Pasadena, California 91125 \\ Henry C. Yuen \\ Department of Fluid Mechanics, TRW Defense and Space Systems Group, Redondo Beach, \\ California 90278 \\ (Received 22 December 1977; final manuscript received 16 May 1978) \\ The stability of two-dimensional infinitesimal disturbances of soliton solutions of the two-dimensional \\ nonlinear Schrödinger equation is considered. Previous results for small transverse wavenumber are \\ extended numerically to arbitrary values.
}

We are interested in the evolution of a weakly nonlinear deep-water wavetrain in two space dimensions. The wavetrain has a carrier wave vector $\mathrm{k}_{0}=\left(k_{0}, 0\right)$ and a carrier frequency $\omega_{0}$, and is subject to slowly varying, two-dimensional modulations in wave vector, frequency, and amplitude. Zakharov ${ }^{1}$ showed that the slowly vary ing envelope of the modulated wavetrain satisfies the two-dimensional nonlinear Schrödinger equation

$i\left(\frac{\partial \psi}{\partial t}+\frac{\omega_{0}}{2 k_{0}} \frac{\partial \psi}{\partial x}\right)-\frac{\omega_{0}}{8 k_{0}^{2}} \frac{\partial^{2} \psi}{\partial x^{2}}+\frac{\omega_{0}}{4 k_{0}^{2}} \frac{\partial^{2} \psi}{\partial y^{2}}-\frac{1}{2} \omega_{0} k_{0}^{2}|\psi|^{2} \psi=0$,

where $x$ and $y$ are horizontal spatial coordinates, $t$ is time, and the complex envelope $\psi$ is related to the elevation $\eta(x, y, t)$ of the free surface by the expression

$$
\eta(x, y, t)=\operatorname{Re}\left[\psi(x, y, t) \exp \left(i k_{0} x-i \omega_{0} t\right)\right] .
$$

For oblique plane modulations, $\psi$ is a function of $z$ and $t$, where $z$ is defined by

$$
z=x \cos \alpha+y \sin \alpha \text {. }
$$

Equation (1) can then be written as

$$
\begin{gathered}
i\left(\frac{\partial \psi}{\partial t}+\frac{\omega_{0}}{2 k_{0}} \cos \alpha \frac{\partial \psi}{\partial z}\right)-\frac{\omega_{0}}{8 k_{0}^{2}}\left(1-3 \sin ^{2} \alpha\right) \frac{\partial^{2} \psi}{\partial z^{2}} \\
-\frac{1}{2} \omega_{0} k_{0}^{2}|\psi|^{2} \psi=0
\end{gathered}
$$

which is the one-dimensional nonlinear Schrödinger equation. For $\alpha<\sin ^{-1}(1 / 3)^{1 / 2}=35.26^{\circ}$, (4) possesses soliton solutions of the form

$$
\begin{aligned}
\psi(z, t)= & b_{0} \operatorname{sech}\left[\frac{k^{2} b_{0}}{\left(1-3 \sin ^{2} \alpha\right)^{1 / 2}}\left(z-\frac{\omega_{0}}{2 k_{0}} \cos \alpha t\right)\right] \\
& \times \exp \left[-(i / 4) \omega_{0} k_{0}^{2} b_{0}^{2} t\right]
\end{aligned}
$$

(see Fig. 1). For $\alpha>35.26^{\circ}$, no steady solutions which decay as $|z| \rightarrow \infty$ exist.

It has been shown by Vakhitov and Kolokolov ${ }^{2}$ that the envelope soliton solution (5) is stable to one-dimensional perturbations independent of the coordinate normal to $z$. We discuss its stability to infinitesimal two-dimensional perturbations. We define

$$
T=-\omega_{0} t, \quad X=2 k_{0}\left(x-\frac{\omega_{0}}{2 k_{0}} t\right), \quad Y=2 k_{0} y, \quad A=\frac{k_{0} \psi}{\sqrt{2}}
$$

and in terms of $A, X, Y$, and $T$, rewrite (1) as

$$
i \frac{\partial A}{\partial T}+\frac{1}{2} \frac{\partial^{2} A}{\partial X^{2}}-\frac{\partial^{2} A}{\partial Y^{2}}+|A|^{2} A=0 .
$$

Without loss of generality, we consider the case of $\alpha$ $=0$, since all other values of $\alpha<35.26^{\circ}$ can be recovered by a simple geometrical scaling. The soliton solution is

$$
A^{(0)}(X, T)=\phi^{(0)}(X) \exp \left(i \gamma^{2} T\right),
$$

where

$$
\phi^{(0)}(X)=\sqrt{2} \gamma \operatorname{sech}(\sqrt{2} \gamma X) .
$$

We examine two-dimensional perturbations of the form

$$
A^{(1)}(X, Y, T)=\phi^{(1)}(X) \exp (i K Y-i \Omega T),
$$

where $A^{(1)}$ is small compared with $A^{(0)}$.

Zakharov and Rubenchik ${ }^{3}$ showed that the real and imaginary parts of $\phi^{(1)}$, denoted by $u$ and $v$, respectively, satisfy the following fourth order, non-self-adjoint eigenvalue problem

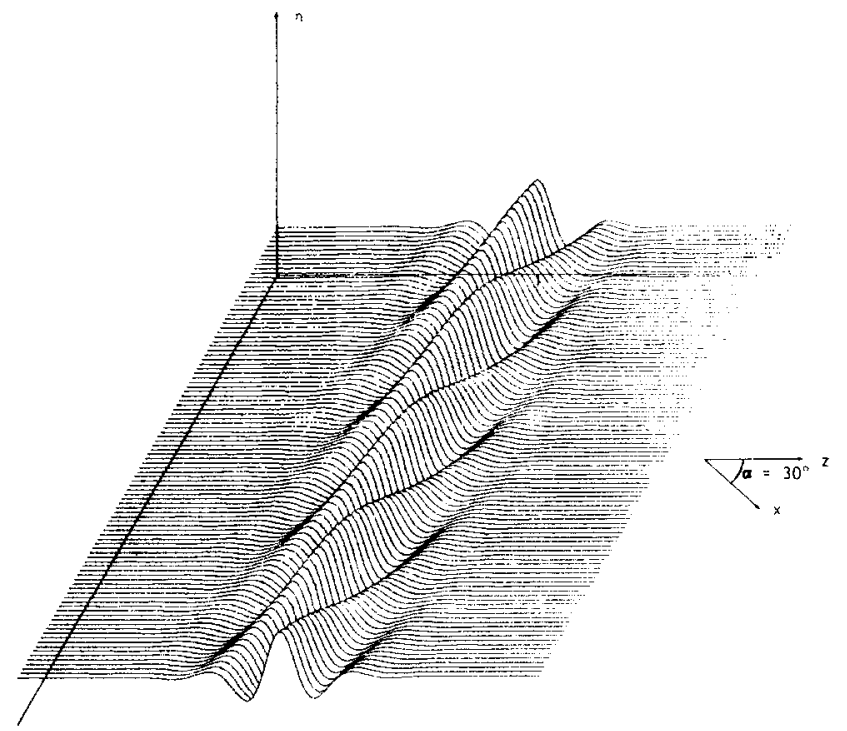

FIG. 1. Free surface corresponding to a plane oblique envelope soliton making an angle of $30^{\circ}$ with the carrier wave vector. 


$$
\begin{aligned}
& \left(L_{0}-K^{2}\right) u=\Omega v, \\
& \left(L_{1}-K^{2}\right) v=\Omega u, \quad-\infty<X<\infty,
\end{aligned}
$$

where

$$
\begin{aligned}
& L_{0}=-\frac{1}{2} \frac{d^{2}}{d X^{2}}+\gamma^{2}-\phi^{(0) 2}, \\
& L_{1}=-\frac{1}{2} \frac{d^{2}}{d X^{2}}+\gamma^{2}-3 \phi^{(0) 2},
\end{aligned}
$$

and the eigenfunctions vanish as $|X| \rightarrow \infty$.

They examined the system analytically for $K$ and $\Omega$ small, and demonstrated by ingenious arguments the existence of two branches of eigenvalues starting from $K=\Omega=0$. These branches correspond to even and odd modes. The even mode is stable with $\Omega^{2}>0$; the odd mode is unstable with $\Omega^{2}<0$. They developed $\Omega^{2}$ as a series in $K^{2}$ and found the coefficients of $K^{2}$ and $K^{4}$. Extrapolation based on the leading terms suggested the existence of a most unstable transverse perturbation wavenumber $K_{\max }$ and a cutoff wavenumber $K_{c}$.

We have extended their results numerically by computing solutions of (10) for arbitrary values of $K$ and have also searched for other possible branches with $\Omega^{2}$ real.

By examining the system (10) for large $X$, we can analytically demonstrate the existence of a continuous spectrum (i.e., improper eigenfunctions) for

$$
\begin{array}{ll}
\Omega^{2}>0, & K^{2}>\gamma^{2}, \\
\Omega^{2}>\left(\gamma^{2}-K^{2}\right)^{2}, & K^{2}<\gamma^{2} .
\end{array}
$$

In the remaining part of the $\left(K^{2}, \Omega^{2}\right)$ plane, we obtain two branches corresponding to those found by Zakharov and Rubenchik, with properties in qualitative agreement with their predictions. We find that for the odd unstable mode

$$
K_{\max }^{2}=0.65 \gamma^{2},|\operatorname{Im} \Omega|_{\max }=0.66 \gamma^{2},
$$

and

$$
K_{c}^{2}=1.18 \gamma^{2} \text {. }
$$

The even stable mode bends back and apparently connects with the continuous spectrum at $K=0$. The results are shown in Fig. 2.

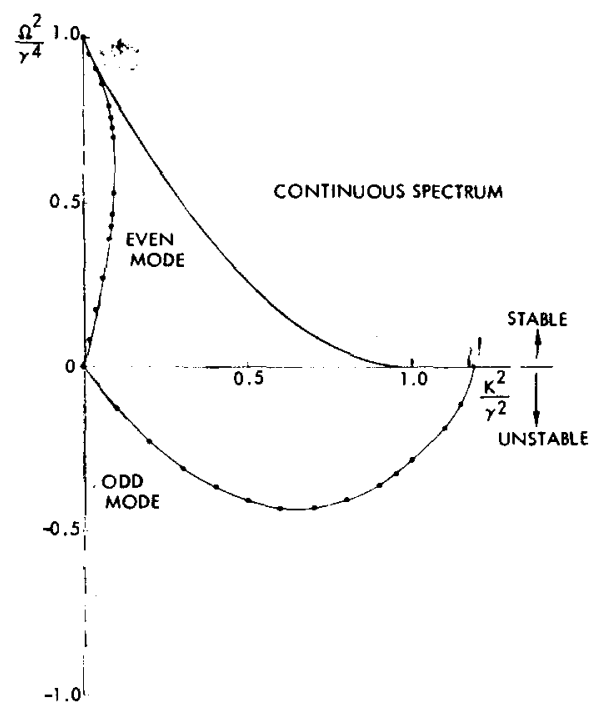

FIG. 2. Stability diagram for a plane soliton subject to infinitesimal two-dimensional perturbations. The stability curves are obtained by joining the numerical results shown by the dots.

Vakhitov and Kolokolov ${ }^{2}$ proved that for $K^{2}=0, \Omega^{2}$ must be real and nonnegative and their results suggested the possible existence of discrete eigenvalues for $K^{2}=0$, different from $\Omega=0$, and branches emanating from these points. Our numerical results indicate that no such discrete eigenvalues exist. The possibility, however, of complex eigenvalues for $K^{2}>0$ cannot be ruled out at present.

This work was sponsored by the Applied Physics Laboratory of The Johns Hopkins University under Contract APL/JHU No. 600613.

${ }^{1}$ V. E. Zakharov, Zh. Prikl. Mekh. Tekh. Fiz. 9, 86 (1968) [J. Appl. Mech. Tech. Phys. 2, 190 (1968)].

${ }^{2}$ N. G. Vakhitov and A. A. Kolokolov, Izv. Vys. Uch. Zav. Radiofiz. 16, 1020 (1973) [Radiophys. Quant. Elect. 16, 745 (1973)].

${ }^{3}$ V. E. Zakharov and A. M. Rubechik, Zh. Eksp. Teor. Fiz. 65, 997 (1973) [Sov. Phys. -JETP 38, 494 (1974)]. 